\title{
The Effectiveness of Mindfulness Based Stress Reduction Intervention on Emotion Regulation Problems and Blood Sugar Control in Patients With Diabetes Type II
}

\author{
Zahra Tavakoli ${ }^{1}$ \& Hamid Kazemi-Zahrani² \\ ${ }^{1}$ MSc, Clinical Psychologist, Department of Psychology, Najaf Abad Azad University, Isfahan, Iran \\ ${ }^{2}$ Assistant Professor, Department of Psychology, Payame Noor University, Tehran, Iran \\ Correspondence: Hamid Kazemi-Zahrani, Department of Psychology, Payame Noor University, PO BOX: \\ 19395-3697, Tehran, Iran. E-mail: hkazemi56@pnu.ac.ir
}

Received: January 2, 2018 Accepted: January 30, 2018 Online Published: February 10, 2018

doi:10.5539/gjhs.v10n3p111 URL: https://doi.org/10.5539/gjhs.v10n3p111

\begin{abstract}
Aim and Background: Diabetes is one of the most prevalent and costly chronic diseases that imposes many limitations on the activities of the patient. Stress reduction treatment based on mindfulness is an intervention which is used in mind-body medicine in order to reduce mental and physical disorders in patients with chronic diseases. So, this study aims to investigate the effectiveness of stress reduction treatment based on mindfulness on emotion regulation problems and glycemic control in patients with type 2 diabetes.

Methods and Materials: The paper is an experimental study based on control and treatment groups with pre-test and post-test. 34 male and female patients with type 2 diabetes having at least high education, from Molasadra Clinic of Isfahan, were selected and were placed randomly in two groups of control $(\mathrm{N}=17)$ and treatment $(\mathrm{N}=17)$. Pre-test stage was done for both two groups by cognitive emotion regulation questionnaire (CERQ) and also by means of Glucometer to measure glycemic of patients. The treatment group for 8 sessions of 2 hours (once a week) was placed under the training of mindfulness-based stress reduction intervention. Afterwards, the post-test was done for both groups. The obtained data using SPSS software version 20 and multivariate analysis of covariance were analyzed.
\end{abstract}

Findings: The findings showed that MBSR had effect on emotion regulation problems, and glycemic control of patients with type 2 diabetes.

Conclusions: On the basis of results, MBSR can have positive impact on emotion regulation problems, and glycemic control of patients.

Keywords: diabetes, mindfulness-based stress reduction, emotion regulation, glycemic control

\section{Introduction}

\subsection{Introduction to the Problem}

Diabetes is a complex metabolic disorder which is characterized by hyperglycemia (high blood sugar). Hyperglycemia is a result of impaired secretion or functioning of insulin or both. Diabetes is a disabling chronic disease (Barnard, Lioyd, \& Holt, 2012). Because of physical problems like retinopathy, nephropathy, neuropathy, cardiovascular diseases and psychological effects, diabetes significantly affects patients' quality of life (Longo et al., 2012).

Physical and mental stresses stimulate neuro-hormonal pathways, specifically hypothalamic-pituitary-adrenal axis. Catecholamine and glucocorticoid axes affect structure and function of specific tissues, resulting in cytokine secretion. All these increase glucagon production and reduce reabsorption or breaking down of sugar in peripheral muscles. Cytokinin basically causes stress oxidative and inflammation processes through interleukin 6, which results in insulin resistance and cardiovascular complications. Relaxation is a process which can regulate cortisol and other stress hormones. Structured meditations, such as stress-based mindfulness, teach how to evoke such a response by focusing on diaphragmatic breathing (Dinardo, 2009). 


\subsection{Importance of the Problem}

Previous studies on psychological problems of diabetes mellitus patients have shown that $40 \%$ of the population had psychological problems. Major complaints have mostly been about prevalence of the disease, depression, obsession-obligation, physical problems, and anxiety and distress. A review study on studies conducted in years 2006 to 2011 in United States and Europe showed widespread comorbidity between depression and diabetes (Roy \& Lioyd, 2012). Depression in diabetes patients is accompanied by self-care behaviors that are lower than the desired level and less compliance to the required medications and diet (Karan, Tanveer, Jaskanwal, \& Jayadave, 2012). Poor emotional health leads to other undesirable outcomes, such as reduced quality of life, undesirable metabolic control, and increased mortality rate (Fisher et al., 2008). There are at least three reasons that justify psychological interventions for diabetes patients: improved disease and situation acceptance by the patient, behavior changes for better self-care, and eliminations of the psychological barriers to disease control (Feifer \& Tansman, 1999).

The relationship between quality of life and psychological health and the prevalence of psychological problems in patients with diabetes necessitate effective interventions to improve psychological health and quality of life for these patients. In this study, MBSR interventions are employed to deal with emotion regulation problems and blood sugar control. When it comes to emotion regulation, it is a cognitive coping strategy that persons use after experiencing negative situations that cause reduce, maintain or increase an emotion. These coping strategies include self-blame, acceptance, rumination, positive refocusing, refocus on planning, positive reappraisal, putting into perspective, catastrophizing and other-blame (Gross, 1998).

Mindfulness is a type of meditation that focuses on conscious presence in the moment in a purposeful and nonjudgmental manner. In the past 30 years, there has been a growing interest in using mindfulness for therapeutic purposes; a review of literature shows more than 70 published papers by 2007 (Ludwig \& Kabat-Zinn, 2008). Stress reduction-based mindfulness is a structured group program with the aim of reducing pain and stress and improving psychological health for patients. Studies have shown that MBSR does not have any negative effects on fibroma, chronic pain, rheumatoid arthritis, diabetes, chronic fatigue syndrome, cancer, blood pressure, aids, skin diseases, multiple chemical sensitivity and cardiovascular diseases. In fact, it has long-term positive therapeutic outcomes in distress reduction in various chronic diseases (Hartmann et al., 2012). Treatment of diabetes, as a worldwide chronic disease, requires constant and effective blood sugar control. Blood sugar control is closely related to stress, since stress causes sugar to be released into blood. Previous studies have investigated the relationship between stress and weak blood sugar control in those with diabetes. They have also investigated the effect of different stress management strategies on blood sugar levels (T. Morris, Moore, \& F. Morris, 2011).

Compared with the general population, patients with diabetes are twice as likely to develop anxiety and depression. This results in less compliance with treatment and therefore weak blood sugar control. Evidence shows that treating psychological issues in those with diabetes can improve their health. Psychological distress is related to impaired blood sugar control in people with diabetes (Karan et al., 2012).

The core of MBSR is to teach mindfulness meditation and its applications in daily life and in coping with stress, pain, and illness (Rosenzweig et al., 2010). Mindfulness-based stress reduction is a behavioral intervention that relies on concentrating on self. MBSR exercises center around focusing on thoughts, emotions, and perceptions. These skills are acquired through focusing on breathing to focusing on every other living activity. MBSR helps by changing people's understanding of stressful events and enhancing their ability to control their life. It has been shown that MBSR has positive effects on physical and psychological symptoms and can be an effective auxiliary treatment for physical illnesses. MBSR results in significant improvements in general aspects of welfare, including quality of life, approaches of dealing with chronic diseases, anxiety, and depression (Grossman, TiefenthalerGilmer, Raysz, \& Kesper, 2007).

Despite the above-mentioned generally-approved positive effects of MBSR, few studies have so far been conducted on the effectiveness of such approaches on chronic diseases like diabetes in Iran. There is no doubt that MBSR is an effective help in this regard. Therefore, the main goal in this study is to investigate the effectiveness of MBSR on emotion regulation and blood sugar control in patients with diabetes type II. To this aim, the study will first introduce this type of intervention, and then try to teach MBSR approach with respect to research variables. Accordingly, the main question is: "Does MBSR have positive effects on emotion regulation and blood sugar control?"

\section{Materials and Methods}

This study is an applied research in nature. Such studies are also called action research and emphasize providing 
specific solutions in the course of real activities.

The study is quasi-experimental and uses control and experimental groups, as well as pre-test and post-test. The intervention was used for the experimental group. No interventions were used for the control group.

Research population consists of patients with diabetes type II who were referred to Mollasadra Diabetes Clinic in Isfahan, Iran. In this study 20 individuals were included in each of the experimental and control groups through directed sample selection (considering loss). At the end, 34 individuals remained that were assigned to two 17-member groups. Inclusion criteria were: absence of any severe psychiatric disease, lack of amputee or maims, being on no psychiatric medications, no experience of diabetic coma, high school diploma or higher, 18 years of age or above, a member of Mollasadra Diabetes Clinic, and not following any other training program before and during MBSR intervention sessions.

Each group was given the Cognitive Emotion Regulation Questionnaire (CERQ) (Garnefski, Kraaij, \& Spinhoven, 2001) and their blood glucose levels (glucometer device), were measured. This was the pretest stage. Afterwards, each group received MBSR in the course of eight sessions (two months, one two-hour session per week). At the post-test stage, the above questionnaire was again filled by the subjects, and their blood glucose levels were measured by researcher who had received the necessary training to measure blood glucose levels.

The CREQ is a self-report questionnaire consisting of 36 items. It is an easy-to-use tool which is applicable for all individuals of age 12 or above (in both normal and clinical populations). The results of Garnefski and Kraaij (2007) showed that the CERQ had good factorial validity and high reliabilities, with Cronbach's as ranging between 0.75 and 0.87 (Garnefski \& Kraaij, 2007). The subscales evaluate nine cognitive approaches: self-blame, acceptance, rumination, positive refocusing, refocus on planning, positive reappraisal, putting into perspective, catastrophizing, and other-blame. Scores span from 1 (almost never) to 5 (almost always). Each subscale includes four items. The overall score for each subscale is calculated by adding up the scores of different items, which means that the score for each subscale will be between 4 and 20. A high score for a subscale means that the related approach is used in most of the cases by the person in the face of stressful and negative experiences.

The results showed that the measure consists of 9 factors, and distribution of test items was consistent with that of the original test. In addition, the correlation pattern observed among subscales of the questionnaire indicate that the questionnaire is multidimensional with every subscale benefitting from a relative independence. In this respect, results of factor analysis in different populations have shown that the 9-factor structure of the CERQ is unchangeable according to sex and age variables (Endler \& Parker, 1993).

To measure blood glucose levels of diabetic patients we used a glucometer device. A glucometer can function using only a modicum of blood (1.5 $\mu$ lit). Rheney and Kirk (2000) showed that there was minimum difference between the results achieved by this device and laboratory data (Rheney \& Kirk, 2000). We used GO-based strips (Glucose Oxidase) in our glucometer device. According to Tang et al. (2001), increasing oxygen pressure in these strips results in decreased measured glucose. However, different pressures of oxygen have a negligible effect on measurements (Tang, Louie, Lee, Miller, \& Kost, 2001).

Ethical considerations: to observe research ethics, MBSR was also applied for the control group in 4-5 sessions after the post-test stage.

Table 1. A brief description of intervention sessions based on Kabat-Zinn instractions

\begin{tabular}{|c|c|}
\hline Sessions & Brief Description \\
\hline \multirow{5}{*}{ Session 1} & 1. Introduction \\
\hline & $\begin{array}{l}\text { 2. Theory: physiology and symptoms of stress, anxiety, and distress, as well as definition of emotion } \\
\text { regulation, self-regulation, blood sugar and blood sugar control. introducing MBSR and a summary of } \\
\text { future activities. }\end{array}$ \\
\hline & $\begin{array}{l}\text { 3. Practice: assessment of symptoms of distress issues using Simons and Gaher's Distress Tolerance } \\
\text { Scale (DTS), emotion regulation using CERQ-P, self-regulation using Miller and Brown's Self } \\
\text { Regulation Questionnaire (SRQ), and measurement of blood sugar levels (fasting) by means of } \\
\text { glucometer. }\end{array}$ \\
\hline & 4. Technique: body scan. \\
\hline & 5. Participants' feedback, after practicing - giving instructions for practicing at home. \\
\hline
\end{tabular}


1. Follow-up: collecting reports of previous week's exercises.

Session 2 2. Theory: effects of meditation on the brain and the grey matter - research evidence.

3. Technique: sitting meditation.

4. Participant's feedback, after practicing the technique - giving instructions for practicing at home.

1. Follow-up: collecting reports of previous week's exercises.

2. Theory: living in the moment, here. What is Yoga? Why Yoga?

Session 3 3. Technique: a few Yoga moves (in accordance with the physical conditions of each participant and the course's environmental conditions).

4. Participants' feedback, after practicing the technique - giving instructions for practicing at home.

1. Follow-up: collecting reports of previous week's exercises.

Session 4 2. Theory: expanding meditation to life.

3. Technique: grape (raisin, ...) meditation. Mindfulness walking meditation.

4. Participants' feedback, after practicing the technique - giving instructions for practicing at home.

1. Follow-up: collecting reports of previous week's exercises.

Session 5

2. Theory: do you like yourself?

3. Technique: rain meditation.

4. Participants' feedback, after practicing the technique - giving instructions for practicing at home.

1. Follow-up: collecting reports of previous week's exercises.

2. Theory: reconciliation with nature.

3. Technique: lake and mountain meditation.

4. Participants' feedback, after practicing the technique - giving instructions for practicing at home.

1. Follow-up: collecting reports of previous week's exercises.

Session 72 Theory: spreading the love.

3. Technique: forgiveness meditation.

4. Participants' feedback, after practicing the technique - giving instructions for practicing at home.

1. Follow-up: collecting reports of previous week's exercises.

Session 8 2. Theory: a conclusion of the lessons of the past two months.

3. Practice: write yourself down exercise; a method for consolidation of mindfulness.

\section{Results}

In this research, qualified individuals were randomly assigned to control $(\mathrm{N}=17)$ and experimental $(\mathrm{N}=17)$ groups, that this number was in both groups, including 9 women and 8 men. In terms of age category, 1 person in the control group and 2 person in the experimental group, were under the age of 26; 2 person in the control group and 3 person in the experimental group, were between 26-35 years old; 4 person in the control group and 3 person in the experimental group, were between 36-45 years old; 4 person in the control group and 4 person in the experimental group, were between 45-55 years old and 6 person in the control group and 5 person in the experimental group, were between 56-65 years old. Also, 6 person in the control group and 5 person in the experimental group, were single and others were married. In addition, in terms of educational level, in control group, 4 person had diploma, 2 person had Assistant, 6 person had BS, 4 person had MSc and 1 person had P.H.D, and in experimental group, 3 person had diploma, 9 person had BS and 5 person had MSc.

Descriptive results of this study include mean and standard deviation. In addition, we used MANCOVA to test research hypotheses. To check the normality of data, Kolmogorov- Smirnov test was used; to check the homogeneity of variances, Leven test was used; and to check the homogeneity of correlations among research variables, box's M test was used. Below are the research hypotheses and the related obtained results.

Using MANCOVA has a number requirements: 1) normality, 2) homogeneity of variances, 3) homogeneity of 
regression slopes, and 4) multicollinearity. All the requirements were carefully analyzed ( $\mathrm{P}>0.05)$ in this study. Normality was checked and verified using Shapiro-Wilk test. Leven and Box's M tests $(F=1.41)$ showed the equality of variances. Considering the significance level of the subscales, it turns out that 6 subscales of 9 subscales, do not have a prerequisite for the equation of variance, but according to the appropriate statistical sample size for the research project and interval scale of measurement tool, can be passed from this prerequisite. Therefore, MANCOVA could be used to analyze the data. Results of Leven test are shown in Table 2.

Table 2. Results of Leven test about the hypothesis of equality of variances of the two groups in the population

\begin{tabular}{lllll}
\hline \multirow{2}{*}{ Variable } & \multicolumn{2}{l}{ Leven Test } & & \\
\cline { 2 - 5 } & $\mathrm{F}$ & First degree of freedom & Second degree of freedom & Significance \\
\hline Self-blame & 2.82 & 1 & 32 & 0.1 \\
Acceptance & 21.90 & 1 & 32 & 0.000 \\
Rumination & 1.20 & 1 & 32 & 0.2 \\
Positive refocusing & 10.43 & 1 & 32 & 0.003 \\
Refocus on planning & 4.49 & 1 & 32 & 0.04 \\
Positive reappraisal & 4.22 & 1 & 32 & 0.04 \\
Putting into perspective & 4.14 & 1 & 32 & 0.01 \\
Catastrophizing & 0.01 & 1 & 32 & 0.9 \\
Other-blame & 0.001 & 1 & 32 & 0.9 \\
Blood sugar control & 6.35 & 1 & 32 & 0.01 \\
\hline
\end{tabular}

Mean and standard deviation of pre-test and post-test scores of emotion regulation approaches and blood sugar control in intervention and control groups are shown in Table 3.

Table 3. Mean and Standard Deviation of pre-test and post-test scores of emotion regulation approaches and blood sugar control for each group

\begin{tabular}{|c|c|c|c|c|c|}
\hline \multirow{2}{*}{ Approach } & \multirow{2}{*}{ Group } & \multicolumn{2}{|c|}{ Pre-test } & \multicolumn{2}{|c|}{ Post-test } \\
\hline & & Mean & SD & Mean & $\mathrm{SD}$ \\
\hline \multirow{2}{*}{ Self-blame } & Intervention & 13.35 & 3.51 & 9.82 & 2.27 \\
\hline & Control & 13 & 3.39 & 13.17 & 3.18 \\
\hline \multirow{2}{*}{ Acceptance } & Intervention & 13.35 & 3.51 & 15.11 & 3.14 \\
\hline & Control & 13 & 3.39 & 9.46 & 1.05 \\
\hline \multirow{2}{*}{ Rumination } & Intervention & 13.47 & & 9.64 & 2.73 \\
\hline & Control & 12.58 & 3.26 & 12.76 & 2.94 \\
\hline \multirow{2}{*}{ Positive refocusing } & Intervention & 7.23 & 1.39 & 14.11 & 2.47 \\
\hline & Control & 7.58 & 1.41 & 7.82 & 1.42 \\
\hline \multirow{2}{*}{ Refocus on planning } & Intervention & 7.76 & 1.71 & 14.88 & 2.32 \\
\hline & Control & 9.82 & 2.32 & 9.58 & 1.73 \\
\hline \multirow{2}{*}{ Positive reappraisal } & Intervention & 7.35 & 1.65 & 14.47 & 2.69 \\
\hline & Control & 11.29 & 2.05 & 10.94 & 1.51 \\
\hline \multirow{2}{*}{ Putting into perspective } & Intervention & 8.29 & 2.17 & 15.35 & 2.93 \\
\hline & Control & 11.41 & 2.18 & 11.05 & 1.95 \\
\hline
\end{tabular}




\begin{tabular}{llllll}
\hline Catastrophizing & Intervention & 13 & 4.30 & 9.64 & 2.82 \\
& Control & 12.23 & 4.49 & 9.88 & 2.59 \\
\hline Blood sugar control & Intervention & 144.58 & 40.78 & 131.41 & 36.92 \\
& Control & 140.41 & 23.81 & 141.41 & 24.50 \\
\hline
\end{tabular}

Table 4 shows the results of MANCOVA for a comparison of emotion regulation approaches between the two groups. As is shown in Table 4, after normalizing the effect of synchronous variables (sex, education, age, and pre-test) on the dependent variable, a significant difference is observed between intervention and control groups for all emotion regulation subscales, except catastrophizing, $(\mathrm{P}>0.05)$. Therefore, the research hypothesis that MBSR affects different aspects of emotion regulation in patients with diabetes type II is confirmed, except catastrophizing.

Table 4. MANCOVA results for scores of emotion regulation subscales in each group

\begin{tabular}{llllllll}
\hline Subscale & Sum & Degree of freedom & Mean & F score & Significance & Eta & Power of test \\
\hline Self-blame & 49.08 & 1 & 49.08 & 22.23 & 0.000 & 0.49 & 0.99 \\
Acceptance & 88.29 & 1 & 88.29 & 15 & 0.001 & 0.39 & 0.96 \\
Rumination & 30.46 & 1 & 30.46 & 6.38 & 0.01 & 0.21 & 0.67 \\
Positive refocusing & 85.21 & 1 & 85.21 & 20.49 & 0.000 & 0.47 & 0.99 \\
Refocus on planning & 75.42 & 1 & 75.42 & 19.66 & 0.000 & 0.46 & 0.98 \\
Positive reappraisal & 58.68 & 1 & 58.68 & 14.55 & 0.001 & 0.38 & 0.95 \\
Putting into perspective & 72.65 & 1 & 72.65 & 13.80 & 0.001 & 0.37 & 0.94 \\
Catastrophizing & 10.99 & 1 & 10.99 & 2.73 & 0.1 & 0.10 & 0.35 \\
Other-blame & 9.23 & 1 & 9.23 & 5.11 & 0.03 & 0.18 & 0.58 \\
\hline
\end{tabular}

Table 5 shows the results of MANCOVA for a comparison of blood sugar control between the two groups. As is shown in Table 5, after normalizing the effect of synchronous variables (sex, education, age, and pre-test) on the dependent variable, a significant difference is observed between intervention and control groups regarding blood sugar control $(\mathrm{P}<0.05)$. Therefore, the hypothesis that MBSR affects blood sugar control for patients with diabetes type II is confirmed.

Table 5. MANCOVA results for the effect of MBSR on blood sugar control

\begin{tabular}{llllllll}
\hline Variable & Sum of squares & Degree of freedom & Mean square & F score & Significance & Eta & Power of test \\
\hline Blood sugar control & 1402.24 & 1 & 1402.24 & 48.03 & 0.000 & 0.63 & 0.999 \\
\hline
\end{tabular}

\section{Discussion}

According to data analysis results provided in the previous section, MBSR does affect emotion regulation in patients with diabetes type II. Here, "emotion regulation" is a term that specifies different cognitive coping strategies that people used after having experienced negative situations, resulting in reduction, maintenance, or intensification of an emotion. This means that, thoughts and cognition play an important role in a person's ability to manage, regulate, and control feelings and emotions after going through a stressful event. Such coping strategies include self-blame, rumination, acceptance, other-blame, catastrophizing, putting into perspective, positive reappraisal, positive refocusing, and refocus on planning. Each of these strategies have their own specific outcomes. Our results are in line with those of Opialla et al. (2015), Goldin, Ziv, Jazaieri, Hahn and Gross (2013), Vago and Silbersweig (2012), Vøllestad, Sivertsen and Nielsen (2011) and Philippe and Gross (2010). It seems that MBSR has been a good stimulating factor for emotion regulation in patients with diabetes type II.

An explanation for the above result could be that MBSR intervention encouraged patients to look at the negative experiences and events from a different perspective, go through negative emotions less intensely, modify their 
emotional reactions, and therefore, experience less discomfort or stress. Diabetes can negatively affect a person's emotions, as was shown in the pre-test stage of this study. The reason might be sought in the effects of this disease on the psychological states of the patient. Among these problems are self-blame, rumination and other-blame, which are sometimes used in an obsessive manner. A decline in acceptance, focusing, and putting in perspective was also observed in these patients.

MBSR interventions offer a new way of confronting with distressful emotions and emotional strategies by emphasizing reflection on thoughts and changing awareness instead of challenging and changing the thoughts (Hartman et al., 2012). Since emotion regulation has several dimensions, mindfulness strategies were designed so that they could affect all of the aspects of the problem. MBSR exercises can affect the cognitive system and information processing through enhancing a person's awareness of the moment by a number of techniques, such as focusing on breathing and focusing on the body. In this way, this method can improve emotion regulation strategies. The three components of this method - moment-to-moment living, awareness, and nonjudgmental presence increase a patient's acceptance and limits self-blame and rumination (Dinardo, 2009).

According to the results of the study, it seems that the body scan technique has had a good effect on patients' focusing. During the MBSR course, patients received clear instructions and exercises to help them understand the meaning of their thoughts, physical senses and internal emotions that together form a complete package. Emotions and thoughts are generally expressed through personal pronouns. For example, feelings are expressed as "my feelings".

MBSR is new method to follow thoughts and emotions by constantly focusing on the body, and analyzing how and where an emotion is manifested in a physical state. This method can be surprisingly beneficial. One way of achieving this is through the body scan technique. Another way is through yoga exercise that help regulate emotions and thoughts in addition to physical training. Techniques such as eating, listening, and watching meditations, etc. teach patients how to let go of their physical senses in every moment when alone. This will prevent them from thinking about themselves and their experiences. This will limit their referring to self-blame and rumination strategies.

Based on Kabat-zinn's findings (2005), it can be explained that Lake and mountain meditation also seems to have a positive effect on the emotion regulation strategies. This technique creates a sense of unification with both the natural and human world, enhancing flexibility of perspective and acceptance in patients. It can be said that, the forgiveness meditation technique, with the aim of loving and creating love, causes a patient to feel important, standing at the center of a love circle in which all loveable people are standing. The patients will learn to forgive and expand this circle little by little.

Also, according to the Kabat-zinn's finding (2005), it can be said that in this research, this techniques seems to have reduced "other-blame" in patients. "Write yourself down" is another helpful technique for regulating unpleasant emotions towards self and family. It motivates a person to accept himself or herself, and find a personal path in life. This in turn will reduce "self-blame". Moreover, this technique changes the focus from the past to the future, and thus, helps patients to set personal goals and identify personal preferences, enhancing "refocus on planning" and "positive reappraisal" in them.

Another major finding of the study is that MBSR has a positive effect on blood sugar control for patients with diabetes type II. Blood sugar control means awareness of blood glycogen levels and reduce of blood glucose levels, which was related to self-management behavior and psychological stress. The result was adherence to proper treatment, reduce of blood glucose levels and regular blood sugar monitoring. These results were in line with those of Van Son et al. (2013), Van Son, Nyklíček, Pop and Pouwer (2011), Morris, Moore and Morris (2011) and Rosenzweig et al. (2007).

According to the Bishop's finding (2002), it can be explained that Techniques of MBSR such as yoga and body-scan exercises not only enhance physical motion and help regulate emotions, but also reduce blood sugar levels (in cases even to normal levels). Daily yoga exercises for at least 30 minutes have shown to be effective in treating conditions such as asthma, diabetes, chronic obesity, cardiac illnesses, and various mental and psychological disorders. Alternating expansion and contraction of the abdominal viscera and increased abdominal pressure in this technique increases blood pressure and causes the liver, the pancreas and other internal organs to work better. Blood sugar level was dropped (to near-normal level) after lunch in most of the patients, resulting in a significant reduction in the required dosage of medicines like insulin. According to the findings of Whitebird, Kreitzer \& O'Connor (2010) and nanda (n.d), it can be explained that it should be mentioned that before MBSR intervention, all of the patients were on diabetes medications and had diet limitations. Although no changes were made to their medications and diet, it seems that MBSR techniques such as yoga, had significant positive effects on 
their life style. Some might argue that yoga is nothing but physical flexibility and mental peace; however, medical studies have shown that yoga can directly affect central nervous system, blood flow, and metabolism.

As a result, According to the research of Whitebird et al. (2010), it can be explained that an obvious improvement in reduce of blood glucose and diabetes and weight loss is observed after MBSR intervention. This does not necessarily mean that a patient can discontinue his or her medications or diet limitations.

\section{Conclusion}

In agreement with our hypothesis, we found that MBSR led to better emotion regulation and lower levels of blood glucose. MBSR interventions seek to establish and reinforce a nonreactive, nonjudgmental approach to thoughts and emotions and to cultivate acceptance through moment-to-moment awareness or "mindfulness." (Shapiro, Schwartz, \& Bonner, 1998). However, in this research, although in both variables (emotion regulation and blood sugar control) the effect sizes were remarkable, in emotion regulation variable, no significant effect could be demonstrated for the catastrophizing subscale.

So, following this research and according to the results of the analysis of collected data, can be used from MBSR intervention to improve mental health and eliminate psychological problems in patients with diabetes type II. It should be noted that this research has been done in Molasadra Diabetes Clinic of Isfahan, therefore, caution should be exercised in generalizing its results to other similar diabetes clinics. Also, the reaction of some people to some of the techniques of MBSR, it is not only different in different societies, but also different from one group to another. Despite the limitations of the study, this study adds to the sparse literature on stress and late diabetes complications and emphasizes the potential of psychosocial interventions. The specific benefit of MBSR is its preventive nature and wide applicability for a variety of symptoms.

\section{Acknowledgements}

The authors thank the Isfahan University of Medical Sciences for helping them implement the research project .It should be mentioned that, part of the present study was discussed as a proposal in the meeting of the research council of the administration for research development, research assessment and coordination of research centers of Isfahan University of Medical Sciences, and was accepted on November 30, 2015. It received the ethical code IR.MUI.REC.1394.2.130 from Isfahan University of Medical Sciences.

\section{Competing Interests Statement}

The authors declare that they have no competing or potential conflicts of interest.

\section{References}

Barnard, K. D., Lloyd, C. E., \& Holt, R. I. G. (2012). Psychological Burden of Diabetes and What It Means to People with Diabetes. Psychology and Diabetes Care, 1-22. https://doi.org/10.1007/978-0-85729-573-6_1

Bishop, S. R. (2002). What Do We Really Know About Mindfulness-Based Stress Reduction? Psychosom Med, 64, 71-83. https://doi.org/10.1097/00006842-200201000-00010

Dinardo, M. M. (2009). Mind-Body Therapies in Diabetes Management: Background and Clinical Problem. Diabetes Spectrum, 22(1), 30-34. https://doi.org/10.2337/diaspect.22.1.30

Endler, N., \& Parker, J. D. A. (1993). Multidimensioal Assessment of Coping. A Critical Evaluation. Journal of Personality and Social Assessment, 58(5), 844-854. https://doi.org/10.1037/0022-3514.58.5.844

Feifer, C., \& Tansman, M. (1999). Promoting Psychology in Diabetes Primary Care. Professional Psychology: Research and Practice, 30(1), 14-21. https://doi.org/10.1037/0735-7028.30.1.14

Fisher, L., Skaff, M. M., Mullan, J. T., Arean, P., Glasgow, R., \& Masharani, U. (2008). A Longitudinal Study of Affective and Anxiety Disorders, Depressive Affect and Diabetes Distress in Adults with Type 2 Diabetes. Diabet Med, 25, 1096-1101. https://doi.org/10.1111/j.1464-5491.2008.02533.x

Garnefski, N. \& Kraaij, V. (2007). The Cognitive Emotion Regulation Questionnaire. European Journal of Psychological Assessment, 23(3), 141-149. https://doi.org/10.1027/1015-5759.23.3.141

Garnefski, N., Kraaij, V., \& Spinhoven, P. (2001). Negative Life Events, Cognitive Emotion Regulation and Emotional Problems. Personality and Individual Differences, 30, 1311-1327. https://doi.org/10.1016/S0191-8869(00)00113-6

Goldin, Ph., Ziv, M., Jazaieri, H., Hahn, K., \& Gross, J. (2013). MBSR vs Aerobic Exercise in Social Anxiety: FMRI of Emotion Regulation of Negative Self-Beliefs. SCAN, 8, 65-72. 
Gross, J. J. (1998). The Emerging Field of Emotion Regulation: An Integrative Review. Review of General Psychology, 2(3), 271-299. https://doi.org/10.1037/1089-2680.2.3.271

Grossman, P., Tiefenthaler-Gilmer, U., Raysz, A., \& Kesper, U. (2007). Mindfulness Training as an Intervention for Fibromyalgia: Evidence of Postintervention and 3-Year Follow-Up Benefits in Well-Being. Psychother Psychosom, 76, 226-233. https://doi.org/10.1159/000101501

Hartmann, M., Kopf, S., Kircher, C., Faude-Lang, V., Djuric, Z., Augstein, F., \& Friederich, H. C. (2012). Sustained Effects of a Mindfulness-Based Stress- Reduction Intervention in Type 2 Diabetic Patients. Diabetes Care, Published online before print, 35(5), 945-947.

Kabat-Zinn, J. (2005). Coming to Our Senses: Healing Ourselves and the World through Mindfulness. New York: Hyperion.

Karan, J. K., Tanveer, C. T., Jaskanwal, S., \& Jayadave, S. (2012). Screening for Anxiety and Depression in Type 2 Diabetes. Endocrine Abstracts, 221-228.

Longo, D., Fauci, A., Kasper, D., Hauser, S., Jameson, J., \& Loscalzo, J. (2012). Harrison's Principles of Internal Medicine (18th ed.). United States: Mc Grow Hill.

Ludwig, D. S., \& Kabat-Zinn, J. (2008). Mindfulness in Medicine. JAMA, 300(11), 1350-1352. https://doi.org/10.1001/jama.300.11.1350

Morris, T., Moore, M., \& Morris, F. (2011). Stress and Chronic Illness: The Case of Diabetes. Journal of Adult Development, 18(2), 70-80. https://doi.org/10.1007/s10804-010-9118-3

Nanda, A. (n.d). Yoga can help cure diabetes; blood sugar. Retrieved December 8, 2017, from https:/www.mokshamantra.com/yoga-for-diabetes-high-blood-sugar/

Opialla, S., Lutz, J., Scherpiet, S., Hittmeyer, A., Jancke, L., Rufer, M., Gross Holtforth, M., Herwig, U., \& Bruhl, A. B. (2015). Neural Circuits of Emotion Regulation: A Comparison of Mindfulness-Based and Cognitive Reappraisal Strategies. European Archives of Psychiatry and Clinical Neuroscience, 265(1), 45-55. https://doi.org/10.1007/s00406-014-0510-z

Philippe, G., \& Gross, J. (2010). Effect of Mindfulness-based Stress Reduction (MBSR) on Emotion Regulation in Social Anxiety Disorder. Emotion, 10(1), 83-91. https://doi.org/10.1037/a0018441

Rheney, C. C., \& Kirk, J. K. (2000). Performance of Three Blood Glucose Meters. Ann Pharmacother, 34, $317-321$. https://doi.org/10.1345/aph.19187

Rosenzweig, S., Greeson, J. M., Reibel, D. K., Green, J. S., Jasser, S. A., \& Beasley, D. (2010). Mindfulness-Based Stress Reduction for Chronic Pain Conditions: Variation in Treatment Outcomes and Role of Home Meditation Practice. Journal of Psychosomatic Research, 68, 29-36. https://doi.org/10.1016/j.jpsychores.2009.03.010

Rosenzweig, S., Reibel, D. K., Greeson, J. M., Edman, J. S., Jasser, S. A., McMearty, K. D., \& Goldstein, B. J. (2007). Mindfulness-Based Stress Reduction is Associated with Improved Glycemic Control in Type 2 Diabetes Mellitus: A Pilot Study. Altern Ther Health Med, 13(5), 36-38.

Roy, T., \& Lioyd, C. E. (2012). Epidemiology of Depression and Diabetes: A Systematic Review. Journal of Affective Disorders, 142, 8-21. https://doi.org/10.1016/S0165-0327(12)70004-6

Shapiro, S. L., Schwartz, G. E., \& Bonner, G. (1998). Effects of Mindfulness-Based Stress Reduction on Medical and Premedical Students. J Behav Med, 21, 581-599. https://doi.org/10.1023/A:1018700829825

Tang, Z., Louie, R. F., Lee, J. H., Miller, E. E., \& Kost, G. J. (2001). Oxygen Effects on Glucose Meter Measurements with Glucose Dehydrogenase- and Oxidase-Based Test Strips for Point of Care Testing. Crit Care Med, 29, 1062-1070. https://doi.org/10.1097/00003246-200105000-00038

Vago, D. R., \& Silbersweig, D. A. (2012). Self-awareness, Self-Regulation, and Self-Transcendence (S-ART): a Framework for Understanding the Neurobiological Mechanisms of Mindfulness. Front Hum Neurosci, 6, 296. https://doi.org/10.3389/fnhum.2012.00296

Van Son, J., Nyklíček, I., Pop, V. J., Blonk, M. C., Erdtsieck, R. J., Spooren, P. F., Spooren, P. F., Toorians, A. W., \& Pouwer, F. (2013). The Effects of a Mindfulness-Based Intervention on Emotional Distress, Quality of Life, and $\mathrm{HbA}(1 \mathrm{c})$ in Outpatients with Diabetes (DiaMind): A Randomized Controlled Trial. Diabetes Care, 36(4), 823-830. https://doi.org/10.2337/dc12-1477

Van Son, J., Nyklíček, I., Pop, V. J., \& Pouwer, F. (2011). Testing the Effectiveness of a Mindfulness-Based 
Intervention to Reduce Emotional Distress in Outpatients with Diabetes (DiaMind): Design of a Randomized Controlled Trial. BMC Public Health, 11(1), 131. https://doi.org/10.1186/1471-2458-11-131

Vøllestad, J., Sivertsen, B., \& Nielsen, G. H. (2011). Mindfulness-Based Stress Reduction for Patients with Anxiety Disorders: Evaluation in a Randomized Controlled Trial. Behav Res Ther, 49(4), 281-288. https://doi.org/10.1016/j.brat.2011.01.007

Whitebird, R. R., Kreitzer, M. J., \& O'Connor, P. J. (2009). Mindfulness-based stress reduction and diabetes. Diabetes Spectrum A Publication of the American Diabetes Association, 22(4), 226.

\section{Copyrights}

Copyright for this article is retained by the author(s), with first publication rights granted to the journal.

This is an open-access article distributed under the terms and conditions of the Creative Commons Attribution license (http://creativecommons.org/licenses/by/4.0/). 